\title{
riccafd
}

Revista Iberoamericana de Ciencias de la Actividad Física y el Deporte

\section{TIEMPO DE PERMANENCIA EN DIFERENTES RANGOS DE INTENSIDAD EN JUGADORES DE FÚTBOL}

\section{TIME SPENT IN DIFFERENT INTENSITY RANGES IN SOCCER PLAYERS}

\begin{abstract}
Parra-Rojas, Nicolás ${ }^{1}$, García-Cantó, Eliseo², Rosa-Guillamón, Andrés ${ }^{3}$.
${ }^{1}$ Doctor por la Facultad de Educación de la Universidad de Murcia (España). Profesor asociado de la Facultad de Educación de la Universidad de Murcia. nicopa2002@hotmail.com

${ }^{2}$ Doctor por la Facultad de Educación de la Universidad de Murcia (España). Profesor asociado de la Facultad de Educación de la Universidad de Murcia. eligar61@hotmail.com

${ }^{3}$ Doctor por la Facultad de Educación de la Universidad de Murcia (España). Consejería de Educación, Juventud y Deportes de la Región de Murcia. andres.rosa@yahoo.es
\end{abstract}

Agradecimientos: Los autores agradecen su colaboración a los jugadores y al cuerpo técnico de La Hoya Lorca Club de Fútbol (Lorca, España).

Código UNESCO: 2411.06 Fisiología del ejercicio.

Clasificación Consejo de Europa: 6. Fisiología del ejercicio.

Correspondencia: García-Cantó, Eliseo. eligar61@hotmail.com

DOI: http://dx.doi.org/10.24310/riccafd.2019.v8i1.5760

\section{RESUMEN}

El objetivo fue analizar el porcentaje de tiempo en diferentes rangos de intensidad según la posición de juego. Estudio no experimental descriptivo relacional, con 18 futbolistas amateurs, de 20-34 años de edad. Durante 71 partidos de liga, se midieron los porcentajes de tiempo en los niveles de intensidad moderada y vigorosa, siguiendo puntos de corte estándar. Los resultados mostraron diferencias en el porcentaje de tiempo al comparar las distintas posiciones de juego. Los jugadores que ocupaban las posiciones de centrocampista, delantero y extremo son los que presentaron un mayor porcentaje de tiempo en el nivel vigoroso en comparación con los que ocupan las posiciones defensivas; siendo estos, los defensas centrales y defensas laterales, los que mostraron un mayor porcentaje de tiempo en moderada intensidad en relación a aquellos. La posición de juego es una de las variables que condicionan el tipo de esfuerzo físico realizado por el futbolista en competición.

Palabras clave: frecuencia cardíaca, rango de intensidad, puesto específico, fútbol. 


\section{ABSTRACT}

The aim was to analyze the percentage of time in different intensity ranges according to the game position. Non-experimental descriptive relational study, with 18 amateur soccer players, aged 20-34 years old. For 71 league games, the percentages of time in moderate and vigorous intensity levels were measured, following standard cut-off points. The results showed differences in the percentage of time when comparing the different game positions. Players who occupied the positions of midfielder, forward and wing-player are those that presented a higher percentage of time in the vigorous level in comparison with those that occupy the defensive positions; being these, central-defenders and lateral-defenders, those that showed a higher percentage of time in moderate intensity in relation to those. Game position is one of the variables that determine the type of physical effort made by the footballer in competition.

Key words: heart rate, intensity range, specific position, soccer.

\section{INTRODUCCIÓN}

El fútbol es un deporte que integra acciones intermitentes de alta intensidad y corta duración, y otras de baja intensidad y mayor duración; por lo que se trata de una disciplina que solicita todos los sistemas energéticos tanto anaeróbicos como aeróbicos ${ }^{1-2}$.

La respuesta cardíaca de los jugadores de fútbol sufre grandes variaciones durante su práctica; siendo un indicador fisiológico fundamental la frecuencia cardíaca, entendida como el establecimiento de un ritmo en latidos por minuto (Ipm) que el futbolista debe utilizar para lograr aquella intensidad deseada ${ }^{3}$.

Para medir el esfuerzo físico resulta fundamental la variable del tiempo de permanencia que los jugadores se encuentran en los diferentes rangos de intensidad ${ }^{4}$. Para medir el tiempo que los jugadores han estado en los diferentes niveles o rangos de intensidad se puede emplear la fórmula de la frecuencia cardíaca de reserva (FC.Res.) propuesta por Karvonen, Kentala y Mustala ${ }^{5}$.

Además de esto, es importante destacar que, con el objetivo de llevar a cabo un análisis de las exigencias físicas y fisiológicas del futbolista, es imprescindible atender a una variable fundamental en la preparación física moderna, que no es otra que el puesto específico ocupado por el futbolista en el terreno de juego, ya que los requerimientos energéticos de cada sujeto serán diferentes en función de la posición ocupada por éste ${ }^{6}$.

También existen otra serie de factores que influyen en el resultado de los registros de la respuesta cardiaca del futbolista tales como las dimensiones del terreno de juego, la evolución del resultado, el planteamiento táctico, las características del terreno de juego, la condición física del jugador, las inter- 
rupciones a lo largo del partido, la inferioridad o superioridad numérica y la hora del día ${ }^{4,7,8}$.

Numerosos estudios sugieren que los niveles de intensidad más frecuentes registrados en futbolistas se encuentran siempre en torno al $80 \%$ con respecto a su frecuencia cardíaca máxima ${ }^{4,9-13}$. En un estudio con jugadores profesionales de baloncesto se obtuvieron resultados de frecuencia cardiaca media muy parecidos a los obtenidos en otros trabajos realizados en fútbol y fútbol sala ${ }^{14}$; es decir, intensidades en el juego por encima del $80 \%$ de intensidad respecto a la frecuencia cardíaca máxima ${ }^{15}$. En otro trabajo, se encontraron resultados parecidos en el deporte del voleibol, con intensidades tanto en centrales como en líberos por encima del $80 \%$ con respecto a su frecuencia cardiaca máxima ${ }^{16}$.

Diversas investigaciones que han analizado la respuesta cardíaca de deportistas en diferentes disciplinas, han observado que esta se encuentra condicionada, especialmente, por la ejecución de las acciones técnicas específicas de competición: baloncesto ${ }^{17-19}$, hockey patines ${ }^{20}$, voleibol ${ }^{21}$, fútbol-sala ${ }^{14}$, e incluso judo 22 .

No obstante, las mediciones realizadas en la mayoría de los estudios no han analizado el tiempo de permanencia en los diferentes niveles de intensidad durante largos periodos de tiempo, lo que dificulta el aporte de hallazgos que permitan el diseño de una preparación del entrenamiento con un enfoque individualizado; pudiendo así optimizar el volumen y la intensidad de trabajo en los entrenamientos y relacionarlos con los de la competición.

En base a lo anterior, el objetivo de este estudio fue analizar el porcentaje de tiempo de permanencia en los niveles de intensidad moderada y vigorosa según la posición ocupada en el terreno de juego en futbolistas amateurs durante los encuentros disputados en las temporadas 2011-2012 y 2012-2013.

\section{MATERIAL Y MÉTODO}

\section{Diseño y muestra}

El estudio se enmarca dentro de un paradigma cuantitativo. Se trata de un estudio empírico, no experimental, descriptivo y relacional ${ }^{23}$. El diseño del muestreo fue de tipo no probabilístico intencional (por conveniencia). Se eligió este club de fútbol por la predisposición positiva de los jugadores y del cuerpo técnico a participar en el estudio.

Se plantearon como criterios de inclusión, tener 18 años y llevar practicando fútbol durante los últimos tres años. Los criterios de exclusión fueron: no haber pertenecido a la plantilla del equipo durante las dos temporadas analizadas; y no haber jugado como mínimo 15 partidos en cada una de las dos temporadas. 
Por tanto, de los 22 jugadores de la primera temporada y los 23 jugadores de la segunda temporada, finalmente participaron 18 futbolistas amateurs, de edades comprendidas entre los 20 y los 34 años, pertenecientes a un club de fútbol militante en el grupo XIII de la tercera división española de fútbol (véase la tabla 1).

En el estudio se respetaron las normas deontológicas reconocidas por la Declaración de Helsinki ${ }^{24}$; se cumplieron las recomendaciones de Buena Práctica Clínica de la $\mathrm{CEE}^{25}$, y la normativa legal vigente española que regula la investigación clínica en humanos ${ }^{26}$.

Tabla 1. Características de la muestra $(n=18)$.

\begin{tabular}{llll}
\hline & Mínimo & Máximo & Media \\
\hline Edad (años) & 20 & 34 & 26,3 \\
Peso $(\mathrm{kg})$ & 64,2 & 85,5 & 73,4 \\
Talla $(\mathrm{m})$ & 1,7 & 1,8 & 1,8 \\
Índice de masa corporal $\left(\mathrm{kg} \cdot \mathrm{m}^{2}\right)$ & 20,5 & 24,9 & 22,5 \\
\hline
\end{tabular}

\section{Variables}

Se analizó la respuesta cardiaca para, posteriormente, medir los porcentajes de tiempo en los niveles de intensidad moderada y vigorosa (véase tabla 2). Estos niveles han sido extraídos de un estudio llevado a cabo por el American College of Sport Medicine ${ }^{27}$; en base a la fórmula de la frecuencia cardiaca de reserva $(F C . \text { Res. })^{5}$ : FC.Res. = FC. Máx. - FC.R., donde FC. Máx. = frecuencia cardiaca máxima, y FC.R. = frecuencia cardiaca en reposo.

Tabla 2. Clasificación de la intensidad de la actividad física en el estudio (extraído del American College of Sport Medicine ${ }^{27}$ ).

\begin{tabular}{lc}
\hline Intensidad & Porcentaje de la frecuencia cardiaca de reserva \\
\hline Muy ligera & $<20$ \\
Ligera & $20-39$ \\
Moderada & $40-59$ \\
Vigorosa & $60-84$ \\
Muy vigorosa & $\geq 85$ \\
Máxima & 100 \\
\hline
\end{tabular}

\section{Instrumentos}

Para la recogida de datos se emplearon un ordenador portátil HP (v.620, Palo Alto, California, EEUU), correas transmisoras de frecuencia cardiaca Suunto Dual Comfort Belt con su correspondiente antena receptora Suunto (Team Pod, Amer Sports, Helsinki, Finlandia), el software Suunto Team Manager y Suunto Monitor (Amer Sports, Helsinki, Finlandia) y Microsoft Excel (v.2010, Redmond, Washington, Estados Unidos). 


\section{Procedimiento}

Se analizaron 71 partidos correspondientes a las temporadas 2011-2012 y 2012-2013. Una vez pedidos todos los permisos correspondientes y habiendo informado a los participantes del proceso a seguir, se llevó a cabo la recogida de datos relativos al porcentaje de tiempo en intensidad moderada e intensidad vigorosa. Los datos fueron registrados por un especialista en preparación física (técnico deportivo en fútbol y licenciado en ciencias del deporte), con formación en el uso de los equipos técnicos.

\section{Análisis estadístico}

Se realizó un análisis descriptivo básico de las variables de estudio. La distribución de las variables resultó no normal al aplicar la prueba de Shapiro-Wilk; por lo que las diferencias entre los grupos se analizaron mediante la prueba no paramétrica $\mathrm{W}$ de Wilcoxon para dos muestras relacionadas. Los datos fueron analizados con el programa Statistical Package for the Social Sciences (v.20.0, Chicago, Illinois, EEUU).

\section{RESULTADOS}

La tabla 3 muestra los descriptivos básicos de la variable tiempo en el nivel de intensidad moderado según el puesto específico en el terreno de juego. Los valores promedio fueron considerablemente superiores en las demarcaciones de defensa central $(24,6 \pm 18,3)$ y defensa lateral $(27,8 \pm 17,9)$. La demarcación de centrocampista es la que presentó valores promedio inferiores $(1,3 \pm 2,4)$.

Tabla 3. Porcentaje de tiempo en el nivel de intensidad moderado entre las distintas posiciones ocupadas en el terreno de juego en el total de partidos de liga.

\begin{tabular}{lcccc}
\hline Posiciones de juego & Mínimo & Máximo & Media & Desviación Típica \\
\hline Defensa central $(n=4)$ & 0 & 71 & 24,6 & 18,3 \\
Defensa lateral $(n=4)$ & 0 & 65 & 27,8 & 17,9 \\
Centrocampista $(n=4)$ & 0 & 18 & 1,3 & 2,4 \\
Extremo $(n=3)$ & 0 & 29 & 6,8 & 6,6 \\
Delantero $(n=3)$ & 0 & 20 & 5,7 & 5,4 \\
\hline
\end{tabular}

Fuente: elaboración propia.

En la tabla 4 se describen los descriptivos básicos de la variable tiempo en el nivel de intensidad vigoroso según el puesto específico en el terreno de juego. En este caso, la demarcación de delantero fue la que mostró valores promedio superiores con respecto a las otras posiciones de juego $(85,0$ $\pm 8,7$ ), especialmente, si se compara con la demarcación de defensa lateral $(63,9 \pm 19,6)$. 
Rev.lb.CC. Act. Fís. Dep. 2019; 8(1): 62-72

Tabla 4. Porcentaje de tiempo en el nivel de intensidad vigoroso entre las distintas posiciones ocupadas en el terreno de juego en el total de partidos de liga.

\begin{tabular}{|c|c|c|c|c|}
\hline Posiciones de juego & Mínimo & Máximo & Media & Desviación Típica \\
\hline Defensa central $(n=4)$ & 0 & 92 & & 18,7 \\
\hline Defensa lateral $(n=4)$ & 0 & 92 & & 19,6 \\
\hline Centrocampista $(n=4)$ & 16 & 99 & & 18,4 \\
\hline Extremo $(n=3)$ & 0 & 95 & & 18,1 \\
\hline Delantero $(n=3)$ & 64 & 100 & & 8,7 \\
\hline
\end{tabular}

Fuente: elaboración propia.

Por último, la tabla 5 muestra las diferencias entre posiciones de juego en el porcentaje de tiempo en moderada a vigorosa intensidad. La prueba W de Wilcoxon detectó diferencias estadísticamente significativas entre defensa central y defensa lateral, defensa central y centrocampista, defensa central y extremo, defensa central y delantero, defensa lateral y centrocampista, defensa lateral y delantero, centrocampista y extremo y extremo y delantero ( $Z$ entre $-8,577$ y $-2,575 ; p$ entre $<0,01 \mathrm{y}<0,001)$.

Tabla 5. Prueba para dos muestras relacionadas mediante la $W$ de Wilcoxon sobre el porcentaje en moderada a vigorosa intensidad entre puestos específicos ocupados en el terreno de juego.

\begin{tabular}{llll}
\hline \multicolumn{1}{c}{ Posiciones de juego } & \multicolumn{1}{c}{ W de Wilcoxon } & \multicolumn{1}{c}{ Z } & \multicolumn{1}{c}{ p } \\
\hline Defensa central $(n=4)$ v. centrocampista $(n=4)$ & 184125,5 & $-6,657$ & $<0,001$ \\
Defensa central $(n=4)$ v. extremo $(n=3)$ & 211252,5 & $-4,425$ & $<0,001$ \\
Defensa central $(n=4)$ v. delantero $(n=3)$ & 173792,5 & $-8,577$ & $<0,001$ \\
Defensa lateral $(n=4)$ v. centrocampista $(n=4)$ & 61987,5 & $-2,575$ & 0,010 \\
Defensa lateral $(n=4)$ v. extremo $(n=3)$ & 104125,5 & $-0,030$ & 0,976 \\
Defensa lateral $(n=4)$ v. delantero $(n=3)$ & 56805,0 & $-4,110$ & $<0,001$ \\
Centrocampista $(n=4)$ v. extremo $(n=3)$ & 96749,5 & $-3,300$ & 0,001 \\
Centrocampista $(n=4)$ v. delantero $(n=3)$ & 59968,5 & $-1,376$ & 0,169 \\
Extremo $(n=3)$ v. delantero $(n=3)$ & 89493,000 & $-5,131$ & $<0,001$ \\
\hline
\end{tabular}

\section{DISCUSIÓN}

El objetivo de este estudio fue analizar el porcentaje de tiempo de permanencia en los niveles de moderada y vigorosa intensidad según la posición ocupada en el terreno de juego (defensa lateral, defensa central, centrocampista, extremo y delantero), en futbolistas amateurs, durante los 71 encuentros correspondientes a las temporadas 2011-2012 y 2012-2013.

Al analizar la respuesta cardíaca de los futbolistas participantes se observó que las posiciones de defensa lateral y de defensa central eran las que mayor 
porcentaje de tiempo permanecía en el nivel de intensidad moderado; mientras que la posición en la que menos porcentaje se registró en este nivel de intensidad fue la de centrocampista. En lo que se refiere al porcentaje de tiempo en el nivel de intensidad vigoroso, se detectó que las posiciones de centrocampista, extremo y delantero eran las que mayor porcentaje de tiempo permanecía en este rango; mientras que la posición en la que menos porcentaje se registra en este nivel de intensidad fue la de defensa lateral.

Esto hallazgos podrían ser debidos al sistema de juego empleado por el equipo, ya que el entrenador primaba la presión tras pérdida del balón en campo contrario; siendo los delanteros y extremos como primera línea, y los centrocampistas como segunda línea, los primeros en realizar la misma; quedando los defensas en posición adelantada de vigilancia.

Los valores del comportamiento de la frecuencia cardiaca de los jugadores objeto de estudio se encontraron la mayor parte del tiempo ( $>72 \%$ del total del tiempo de partido) en el rango de intensidad vigoroso (60-84\% de la FC.Res.). En este sentido, otros trabajos han registrado valores de intensidad mínimos del $77 \%$ y máximos del $87 \%$; lo que quiere decir que la totalidad de los datos obtenidos se corresponde con el rango de intensidad vigoroso ${ }^{4,11-12}$. Sin embargo, en un estudio realizado con jóvenes jugadores de soccer se obtuvieron unas intensidades máximas de hasta el $90 \%$ de la FC. Res., lo que estaría por encima del rango de intensidad vigoroso ${ }^{13}$. Resultados similares a los reportados en disciplinas deportivas que se desarrollan en espacios de juego con menores dimensiones, donde son más frecuentes las acciones de ataque, contraataque y repliegue; lo que provoca porcentajes de tiempo elevados en vigorosa intensidad; como sucede en este caso en el deporte del fútbol-sala ${ }^{14}$.

Los resultados de estos estudios muestran que el fútbol presenta unas demandas condicionales concretas, aunque similares en muchas acciones a otras disciplinas deportivas, que determinan en consecuencia el metabolismo energético del jugador ${ }^{1-2}$; pudiéndose deducir que las acciones explosivas y de breve duración (metabolismo anaeróbico aláctico y láctico) se encuentran asociadas directamente a las situaciones de mayor importancia en la competición.

Por otro lado, en el análisis de los valores promedio registrados en el porcentaje de tiempo en el rango de intensidad vigoroso según el puesto específico, se observó que los laterales presentaron un 63,9\%, los defensas centrales un $65,1 \%$, los centrocampistas un $73,7 \%$, los extremos un $74,7 \%$ y los delanteros un $85 \%$. Estos datos muestran que, a lo largo de un partido de liga, los futbolistas se encuentran mayormente en este rango de intensidad independientemente del puesto ocupado en el terreno de juego, lo que coincide con las medias obtenidas en los estudios señalados anteriormente.

En este sentido, en un estudio 4 llevado a cabo con seis futbolistas profesionales en partidos de pretemporada, se registraron datos de porcentajes con respecto a la frecuencia cardíaca de reserva, que son los siguientes: en los jugadores que 
ocupaban puestos defensivos se obtuvo un porcentaje del $82 \%$ de intensidad, mientras que los centrocampistas y delanteros obtuvieron registros medios del $84 \%$ y $77 \%$ respectivamente. A partir de ahí, si se establece un paralelismo entre el porcentaje de tiempo en el rango de intensidad vigoroso del presente estudio y la media del porcentaje de intensidad con respecto a la frecuencia cardiaca máxima del estudio de García ${ }^{4}$, se puede observar que en el presente trabajo las intensidades más bajas aparecen en los registros de los defensas, tanto centrales como laterales; mientras que en el estudio de García ${ }^{4}$, los niveles más bajos de intensidad se encontraron en el puesto de delantero; datos que coinciden con otro estudio ${ }^{11}$, en el que también se describe la monitorización de la frecuencia cardíaca por puestos específicos obteniendo que, en el caso de los defensas se registró un porcentaje entre el $80 \%$ y el $85 \%$, los centrocampistas el $87 \%$, y los delanteros el $76 \%$.

No obstante, los resultados del presente estudio en relación a los jugadores que ocupan posiciones defensivas no coinciden con datos obtenidos en una investigación en la que se estudió la respuesta cardíaca de jugadores juveniles brasileños ${ }^{28}$, encontrando que en este caso son los defensas los que presentan la mayor cantidad de tiempo en intensidades más altas (entre $95 \%$ y 100\%).

Este estudio destaca por el análisis de un parámetro muy importante en el rendimiento de un tipo de deportistas, de una modalidad colectiva, de una categoría deportiva cercana al ámbito profesional, mediante un procedimiento objetivo, durante un largo periodo de tiempo. El hecho de que estudio se haya llevado a cabo en partidos oficiales es algo poco habitual, ya que existen muchas restricciones a la hora de aplicar una investigación de este tipo a una situación de competición real; lo que no quiere decir que estos resultados no sean replicables por otras investigaciones similares. Sin embargo, tras la realización de este trabajo diversos aspectos permanecen aún por ser aclarados en esta cuestión. No se han analizado variables determinantes del estado de condición física como el porcentaje de masa muscular y de masa grasa, la fuerza muscular y la resistencia aeróbica; así como otras variables como la distancia recorrida, la velocidad de carrera y las aceleraciones. Tampoco se han registrado datos relativos a la respuesta cardiorrespiratoria como el nivel de lactato en sangre, el consumo máximo de oxígeno, la frecuencia cardiaca media o el pico máximo de frecuencia cardiaca. También sería un complemento interesante el uso de cuestionarios de esfuerzo percibido, así como variables como la metodología del entrenamiento y los modelos de juego. Por tanto, se confirma la necesidad de realizar estudios que contemplen estas variables con la finalidad de aportar luz a la problemática de estudio; estudios experimentales, de control aleatorizado, diseño longitudinal, con mayores muestras de estudio.

\section{CONCLUSIONES}

Los resultados de este estudio sugieren la existencia de diferencias en el porcentaje de tiempo en moderada y vigorosa intensidad al comparar las distintas 
posiciones o demarcaciones de juego. Los jugadores que ocupaban las posiciones de centrocampista, delantero y extremo son los que presentaron un mayor porcentaje de tiempo en el nivel de vigorosa intensidad en comparación con los que ocupan las posiciones defensivas; siendo estos, los defensas centrales y defensas laterales, los que mostraron un mayor porcentaje de tiempo en moderada intensidad en relación a aquellos. Al comparar el porcentaje de tiempo de moderada a vigorosa intensidad, los resultados indican la existencia de diferencias entre todas las posiciones de juego, sobre todo entre defensa central y delantero, excepto entre defensa lateral y extremo y centrocampista y delantero.

\section{APLICACIONES PRÁCTICAS}

La posición en el terreno de juego se muestra como una de las variables que condicionan el tipo de esfuerzo físico realizado por el futbolista en la competición. Este hecho podría deberse al sistema de juego empleado por el equipo. Por consiguiente, la preparación tanto física como técnica y táctica debe diseñarse según los principios y los subprincipios de juego, ya que de ello depende en gran parte el rendimiento individual y colectivo.

\section{REFERENCIAS BIBLIOGRÁFICAS}

1. Bangsbo J. Physiology of soccer- with special reference to intermittent exercise. Acta Physiol. Scand.1994; 619(Suppl.): 151.

2. Fandiño L. La fatiga en fútbol. Mecanismos de producción y medios de recuperación. Futbol táctico.2012; 159-167.

3. Zabala M. La frecuencia cardiaca y la regulación del esfuerzo. Apuntes para entrenadores de ciclistas de la Federación Andaluza de ciclismo. Granada: Federación Andaluza de Ciclismo; 2007.

4. García O. Estudio de la frecuencia cardiaca del futbolista profesional en competición: un modelo explicativo a partir del contexto de la situación de juego. Tesis Doctoral. A Coruña: Universidad de A Coruña; 2005. 276p.

5. Karvonen MJ, Kentala E, Mustala O. The effects of training on heart rate; a longitudinal study. Ann Med Exp Biol Fenn.1957; 35: 307-315.

6. Bloomfield J, Polman RCJ, O’Donoghue PG. Physical demands of different positions in FA Premier League soccer. J Sports Sci. 2007; 6: 63-70.

7. Vizcaíno D. Demandas Fisiológicas en Actividades Fitness y Determinación de las Intensidades. INEFC Lérida: Generalidad de Catalunya; 2013.

8. Muñoz J, Castillo D, Yanci J. Análisis de la percepción subjetiva del esfuerzo diferenciado y de la fatiga en distintos formatos de juegos reducidos en fútbol. Rev. Ib. CC. Act. Fís. Dep. 2018; 7(2): 14-28.

9. Ceballos-Gurrola O, Moreno-Villarreal DU, Ochoa Ahmed FA, López-Gonzáles DE. Control de la frecuencia cardíaca durante la competición en el fútbol profesional. Revista de Ciencias del Ejercicio FOD. 2011; 7: 135-138. 
10. García-Mateos J. Análisis y comparativa del comportamiento de la frecuencia cardíaca en entrenamientos de diferente intensidad en fútbol. Trabajo de fin de grado en ciencias de la actividad física y del deporte. Universidad de León; 2015.

11. Ramírez S, Vilaplana J. Descripción de la monitorización de la frecuencia cardíaca en una competición en fútbol. AB Futbol. 2011; 55: 46-90.

12. Aşçi A. Heart Rate Responses during Small Sided Games and Official Match-Play in Soccer. Sports. 2011; 4:31. doi:10.3390/sports 4020031.

13. Barbero-Álvarez JC, Pedro RE, Nakamura FY. Validity of a repeated-sprint ability test in young soccer players. Science \& Sports. 2013; 28(5): 127-131. doi: 10.1016/j. scispo.2012.12.003.

14. Barbero JC, Vera J, Hermoso, VM. Análisis de la frecuencia cardíaca durante la competición en jugadores profesionales de fútbol sala. Apunts de Educación Física y Deportes. 2004; 77: 71-78.

15. Vaquera A, Refoyo I, Villa JG, Calleja J, Rodríguez-Marroyo JA, García-López J, Sampedro J. Análisis de la frecuencia cardiaca en jugadores profesionales de baloncesto. Journal of Human Sport and Exercise. 2008; 3(1): 1-9.

16. Portela-Pozo Y, Dueñas-Nuñez MV, Rodríguez-Stiven E. Estudio de la frecuencia cardiaca durante la competición de voleibol a los jugadores líberos y los centrales. Revista digital Medicina del deporte. 2011; 26: 8-29.

17. Colli R, Faina M. Investigación sobre el rendimiento en basket. Revista de Entrenamiento Deportivo. 1987; 1(2): 3-10.

18. Dal Monte A, Gallozi C, Lupo S, Marcos E, Menchinelli C. Evaluación funcional del jugador de baloncesto y balonmano. Apunts. Medicina de L'Esport. 1987; 94: 243-252.

19. Zaragoza J. Baloncesto: conclusiones a partir del análisis de la actividad competitiva. Revista de Entrenamiento Deportivo. 1996; 10(2): 21-27.

20. Blanco A, Enseñat A, Balagué, N. Hockey sobre patines: niveles de frecuencia cardiaca y lactacidemia en competición y entrenamiento. Apunts: Educación Física y Deportes. 1994; 36: 26-36.

21. Moras G, Zurita C. Valoración de la intensidad del entrenamiento mediante la frecuencia cardiaca en el voleibol. Apunts: Educación Física y Deportes. 1999; 55: 77-84.

22. Dopico J, Iglesias E. Propuesta de utilización simultánea de video y pulsómetro en la caracterización del esfuerzo en Judo. Recursos de actuaciones metodológicas para la enseñanza del entrenamiento, la gestión y organización de la actividad física y del deporte. Junta de Castilla y León: INEF Castilla y León; 1997.

23. Thomas JR, Nelson JK. Métodos de investigación en actividad física. Badalona: Paidotribo; 2007.

24. World Medical Association. Declaration of Helsinki. JAMA. 2013; 310(20): 2191-2194. Disponible en https://www.wma.net/wp-content/uploads/2016/11/DoH-Oct2008.pdf.

25. Comisión Europea. Recomendaciones de Buena Práctica Clínica de la CEE. Documento 111/3976/88 de julio; 1990.

26. Real Decreto 561/1993, de 16 de abril, por el que se establecen los requisitos para la realización de ensayos clínicos con medicamentos. B.O.E. n. ${ }^{\circ} 114$, de 13 de mayo. Madrid: Ministerio de Sanidad y Consumo; 1993. 
27. Garber CE, Blissmer B, Deschenes MR, Franklin BA, Lamonte MJ, Lee IM, Nieman DC, Swain DP. American College of Sports Medicine position stand. Quantity and quality of exercise for developing and maintaining cardiorespiratory, musculoskeletal, and neuromotor fitness in apparently healthy adults: Guidance for prescribing exercise. Medicine and Science in Sports and Exercise. 2011; 43(7): 1334-1359. doi: 10.1249/MSS.0b013e318213fefb.

28. Coelho DB. Determination of the activity's intensity of soccer players during official match play according to the heart rate monitoring. Dissertation. Belo Horizonte-Brasil: Universidade Federal de Minas Gerais; 2005. 